\title{
Research on micro governance path of high- level professional group construction
}

\author{
Guangjiu Chen* \\ Luzhou Vocatinal and Technical College, Luzhou, China
}

\begin{abstract}
Under the background of "double high plan", higher vocational colleges combine the industrial background, strengthen the connection between industrial chain and professional chain. Therefore, colleges and universities delegate power to secondary colleges. Secondary colleges independently improve the grass-roots governance mechanism according to the logic of professional groups, which will help to optimize the power allocation mode, and strengthen the construction of professional organizations. The functions of professional resource allocation, power distribution and operation mechanism, so as to provide management guarantee for the construction of high-level professional groups.
\end{abstract}

Keywords: Build the institute with the group, High level professional group, Micro governance.

\section{Introduction}

The medium and micro-governance is an important part of the governance system and capacity building in colleges and universities. Under the background of "double-high Plan", higher vocational colleges should strengthen the connection between industry chain and specialty chain, and carry out the internal management of "building a college with a group". At this point, colleges and universities will delegate power to the departments to relieve the pressure of "shoulder-to-shoulder" work. The departments will construct a reasonable form of teaching organization and management based on the industrial chain and independently improve the grass-roots governance mechanism according to the logic of professional groups, help to achieve the effective training of professional personnel.

\section{Basic viewpoints and theories of internal governance in colleges and universities}

Since the 1980s, under the guidance of the New Public Management Movement, globalization and Localization of higher education have become the two main development directions of Western universities. On the one hand, pursuing the goal of building a WorldClass University, strengthening the important position of the University in the global Comprehensive National Power competition, on the other hand, serving the important goal

*Corresponding author: 330115925@.qq.com 
of the Intellectual Economy, the university gradually moves from the "marginal position" to the "central area", and becomes one of the core driving forces to promote the national social and economic development.

\subsection{The department is an important place for management}

Foreign scholars point out that the grass-roots department should be an important place for teachers to participate in university governance and the cornerstone of university management (Birnbaum 1991, Benjamin \& amp. In the research of the relationship between university governance structure and external environment, how does the governance structure of university's Internal Faculty, College, School and Department adapt to the change of external environment, and adjust the governance structure accordingly, there is an obvious tendency of strengthening the administrative and efficiency orientation, squeezing the decision-making participation and academic autonomy of the grass-roots departments and colleges, weakening the governance role and function of the departments and colleges. Under the new public management (NPM) movement-oriented university governance, facing the increasingly strong external environmental pressure, the university is no longer simply pursuing academic truth-seeking ivory tower (Mok, 2013). At the same time, special expertise and academic freedom shape unique organizational characteristics and cultural traditions (Christensen, 2011).

\subsection{The idea of professional group construction and its internal management}

The theory of Discipline Connection emphasizes that the specialty cluster in higher vocational colleges should be based on the dominant specialty and discipline foundation (Yuan Hongzhi, 2017). The school teaching resources disposition and the Teaching Organization reality are the foundation which the specialized group establishes, the Teaching Organization stratification plane based on the school reality, through the discipline interior or the neighboring discipline group, reduces the teaching organization each kind of system cost, such as the cost of governance within the Teaching Organization, the cost of teacher management and so on (Mei yaming, 2018). Higher internal governance costs due to management coordination costs in hierarchical organizations (Wang Chunxu, 2018). It can be seen that the form of teaching organization based on the construction of specialty group has both the attribute of market transaction and the attribute of internal management.

\subsection{Reform of teaching organization in higher vocational colleges}

The Different Group logic of specialty group Construction Has Different Influence on Specialty Group and its Teaching Organization (Zhu Jun, 2020); The logic of professional group should include the logic of high-end industrial relations, the logic of high-end industrial form, the logic of post group, the logic of talent training and the logic of professional composition (Deng Ziyun, Zhang Fanping, 2020); The paper also points out that higher vocational schools can deepen the top-level design of professional cluster based on the technology chain, reorganize the resources in the cluster according to the cluster logic, and advance the system guarantee work according to the industry cluster, post cluster and inter-cluster relation (Liu Xiao, 2020). Thus, the focus of the construction of professional groups is organizational construction, the key path is to improve the degree of organization. The above research has provided the theory foundation for this article, simultaneously also has the insufficiency. 


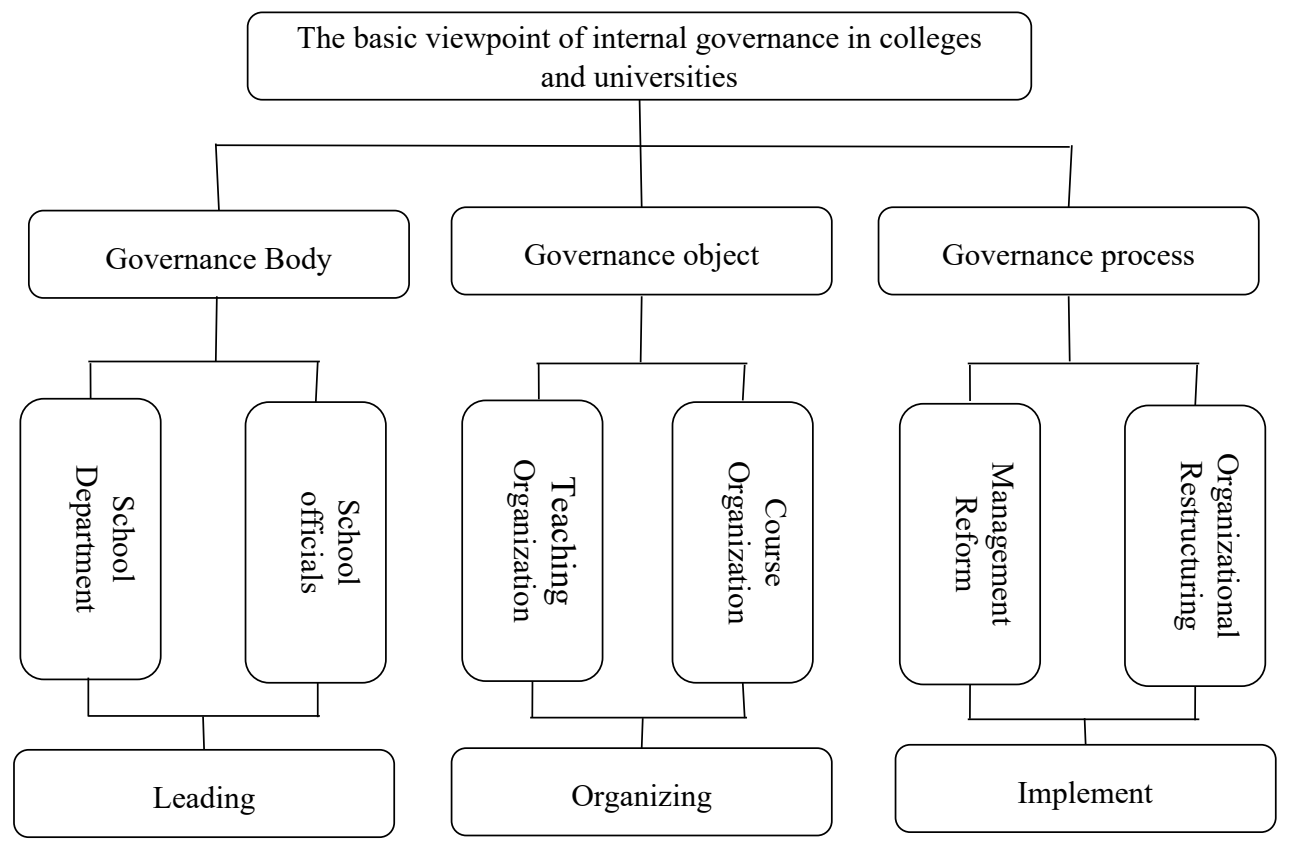

Fig. 1. Internal Governance system of colleges and universities.

\section{The organizational background and implementation dilemma of "building a college with a group of Students"}

Taking the pre-school education major group as an example, since the 18th National Congress of the CPC put forward the idea of "running pre-school education well" , a series of new policies at the national level, including the State Council's "opinions on deepening reform and standardized development of Preschool Education" in November 2018 and the State Council's "guiding opinions on promoting the development of care services for infants and young children under the age of three" in April 2019, have raised preschool education to an unprecedented level, it also expands the connotation of Preschool Education in the vertical age range and Horizontal Content Range, and highlights the idea of "preschool education professional group" . The construction of high-level professional groups in the "double-high plan" launched in 2019 is the concrete implementation of this concept.

\subsection{The background and group logic of "building a college with a group"}

Based on this, the logic of the high-level preschool education major group in our university is mainly based on three aspects: First, the same industry background, job succession. All the specialties in the group belong to the field of teacher education, which is connected with the basic education education service industry. Pre-school education pays equal attention to protecting and teaching, English education in primary schools and Chinese education in primary schools are mainly education, while music education runs through the whole process of basic education. Second, the discipline accomplishment is similar, the basic curriculum is similar. The specialties in the group belong to the education category, and the basic accomplishment of the subjects is similar, which is beneficial to the construction and sharing of resources. The curriculum resources of educational theory such as psychology, pedagogy and teachers' skill such as teachers' spoken language and teachers' calligraphy 
can be built and shared. Third, the two-division team co-construction, practice base sharing. All the teachers in the group have the background of normal education, share highly, and have a reasonable structure, sufficient quantity and high quality double-teacher team. All majors in the group build and share microteaching training room, dance room and other training room, and share out-of-school practice and employment base such as primary school, kindergarten and infant and child care service organization.

\subsection{Implementation dilemma}

The construction of the existing professional groups is based on the linear action logic of "industry as a profession -- Employment", which neglects the complexity of the labor market and needs to be improved into the action logic of "double linkage" (external linkage, internal linkage). The main reason is that, in the concrete practice of specialty group construction, the new group logic has broken the original structure of the secondary colleges, although it is conducive to stimulating new vitality, at the same time, because of the inertia of specialty construction.

However, under the construction of high-level Specialty Group, the second-level college management faces many difficulties. Because of the different group logic, there is a dilemma of cooperation of different specialties in the group-based college construction, and the dilemma of Professional Group Organization has a series of effects on the governance of the second-level college. First of all, from the perspective of the Organization of highlevel professional groups, the organization mechanism of the lack of autonomy in microgovernance of second-level colleges needs to be further deepened, the challenges faced by the second-level college governance have yet to overcome the constraints on the realization of the Organization of high-level Preschool Education professional groups. Secondly, under the administrative-oriented governance structure and the administrative pattern of specialty construction, it is necessary to construct the specialty organization setting, administrativeoriented organizational characteristics and governance structure from the high-level Preschool Education Specialty Group to the second-level College, in order to find out the corresponding integration strategy in the concrete practice, we should have a deep understanding of the inter-embedding mechanism of the dilemma, such as "no boundary", "fragmentation" and "difficult evaluation" .

Based on the two-level college governance reform and the construction of high-level professional group elements, there is a simplified interpretation and negative impact on the two-level college governance reform, it leads to the value misunderstanding, practical problems and performance dilemma in the construction of high-level Specialty Group. Overemphasizing the input of high-level major group elements and ignoring the organizing function of major group resources leads to the deviation of major group elements and the weakening of basic organization efficiency. The non-organization of specialty group construction weakens the efficiency of resource allocation of secondary colleges, it mainly includes: The multi-influence of the unitary specialty construction and the crosscomplement of the specialty group, the bureaucratic organization structure and the bureaucratic operation, and the decentralization of the resource center of the specialty group construction on the resource allocation of the second-level colleges. The "virtualization" of Specialty Group Construction and the dilemma of the second-level college governance performance mainly include: the restriction of "project attachment" and "administration" of the investment of human, financial and policy resources in specialty group construction on the innovation of the second-level college governance, this kind of "project attachment" and "administration" makes it difficult to evaluate the construction output of high-level specialty group. 


\section{The organizational background and implementation dilemma of "building a college with a group of Students"}

\subsection{Micro-governance logic of specialty group construction}

Taking the construction of pre-school education major group as an example, this study analyzes the organizational mechanism of "Building College by Group" and reveals that College Deans Face the dilemma of unequal power and responsibility between their superiors, party secretaries, teachers and themselves, based on this logic, this paper explores the system innovation of micro-governance in higher vocational colleges under the construction of High-level Pre-school Education Specialty Group.

From the analysis of the functions of micro-governance in the construction of professional organizations, the allocation of professional resources, the distribution of power and the operational mechanism, the organizational background and governance logic of "building a college with a group" under the construction of high-level preschool education professional groups, the main points of view: (1) the external governance and internal governance of colleges and universities are two dimensions. The former focuses on the power structure of extraversion at the macro-institutional level, which is easy to induce the one-dimensional extreme governance pattern of external control, weakening the internal motive force and the professional authority of the Dean of the Secondary College. (2) the medium and micro-governance is an important part of the governance system and capacity building of universities. According to the logic of pre-school education group, the secondlevel colleges should construct a reasonable teaching organization and management form based on the industrial chain, and improve the grass-roots governance mechanism independently, in order to achieve pre-school education personnel effective training.

\subsection{The realistic path of micro-governance in specialty group construction}

In order to solve the problem of insufficient autonomy in micro-governance of second-level colleges in the construction of high-level specialty clusters, strengthening the microgovernance efficiency first needs the decentralization of power from the school level to the second-level colleges, the current suggested path is: "to set herself as if she could be held accountable" .

(1) Delegation of authority.The high-level Specialty Group undertakes the function of talent training and industry docking in the new era. The construction of high-level Specialty Group Must Endow the second-level college with certain power in order to fulfill its duty. First of all, under the background of "building colleges by groups", the main goal of higher vocational colleges is to promote the construction of high-level vocational colleges through the construction of high-level professional groups, and finally realize the construction goal of "double-high plan" . Therefore, in the overall management of "building a college with a group of Students", higher vocational colleges must speed up the transformation of their functions, give secondary colleges the power they should have in the construction of highlevel professional groups, curriculum development and other aspects, especially the secondlevel college internal governance has a certain degree of autonomy..

(2) Enablement.In the construction of high-level Specialty Group, we should not only strengthen the ability of microcosmic management of the secondary college, but also dispose all kinds of power. First, academic power should be strengthened. The academic affairs in the construction of high-level specialty group generally involve curriculum development research, Teaching Reform Research, specialty establishment research, enrollment and market, so it should have the autonomy of academic research. Therefore, 
teachers in higher vocational colleges should not only do well in teaching work, but also strengthen research work. Secondly, administrative power should be weakened. In the process of high-level specialty group construction, the Administrative Affairs of the second-level college include Asset Management, fund management, Scientific Research Project Management, personnel management, etc. . In order to deal with the administrative affairs of the Higher Vocational College, the school must have certain administrative power, which comes from the authorization of the school.

(3) Clear responsibility.In the construction of high-level Specialty Group, we should not only empower and empower the second-level college, but also make clear its governance responsibility. Only in this way, the construction of high-level professional groups can achieve the same rights and responsibilities, and the concrete implementation of its microgovernance measures is realistic and sustainable. From the point of view of evaluation index system, it must include content indicators (such as rules, regulations, standards, etc.), process indicators (enrollment rate, graduation rate, professional qualification acquisition rate, etc.) and efficiency indicators (funding, personnel, equipment input and teaching achievement awards, projects, paper output, etc.) . From the perspective of evaluation subject, schools, society and enterprises should take part in evaluation from the perspective of high-level Specialty Group Construction.

\section{Conclusion}

This paper explores the organization logic between the construction of the specialty group and the micro-governance of the second-level college at the medium and micro-level, establishing the institutional link between the construction of specialty clusters and the reform of micro-and medium-scale governance. In order to improve the management level and ability of higher vocational colleges, a systematic study is carried out on the micromanagement under the construction of high-level professional groups at the medium and micro levels, to provide the theoretical basis for promoting the reform of micro-governance in higher vocational colleges.

This paper is a project funded by the research center of Sichuan Education Development, a key research base of philosophy and social sciences in Sichuan Province. It is a micro-governance innovation research on the construction of pre-school Education Professional Group under the background of "Double High Plan" (CJF21054).(2)"research on the guarantee system for the construction of high-level Specialty Group" (LZYGCC202112), a project funded by the scientific research of high-level talents in Luzhou Vocational and Technical College.

\section{References}

1. Song Chaoli. Innovation of leadership concept in the vision of micro-governance modernization [ J ] . Leadership Science, 2015(02) : 50-51.

2. Tan raised an army. Connotation and practice of high-level specialty group construction under type education. Laboratory techniques and management, 2021,38(06) : 237-240 + 253 .

3. Wu Xuehui, Su Xiaoli, Wu Rulian. An analysis of the characteristics of high-level higher vocational colleges and their specialty clusters -- Taking the construction unit of "double-high plan" as an example [ j ] . Journal of Educational Sciences, 2021(06) : 105-111.

4. Li Deneng. A study on the practice of constructing high-level specialty cluster in preschool education--Taking Pingliang vocational and technical college as an example 
[ a ] . Basic Education, Ministry of Education. Proceedings of the 2021 symposium on classroom teaching reform [ $\mathrm{C}$ ] . Basic Education: Basic Education, Ministry of Education, 2021:3.

5. Xu Xingui. Concept Innovation and system reform of micro-governance of unit $[\mathrm{J}]$. Leadership Science, 2014(07) : 8-10. 